\title{
On envy-free perfect matching
}

\author{
C. Arbib ${ }^{a}$, O.E. Karaşan ${ }^{b}$, M.Ç. Pınar ${ }^{\text {b,* }}$ \\ a University of L' Aquila, Italy \\ b Bilkent University, Turkey
}

\section{A R T I C L E I N F O}

\section{Article history:}

Received 27 March 2017

Received in revised form 3 January 2018

Accepted 10 March 2018

Available online 5 April 2018

Dedicated to Martine Labbé on the occasion of her birthday

\section{Keywords:}

Envy-free pricing

Walrasian equilibrium

Perfect matching

Assignment

Shortest path problem

\begin{abstract}
A B S T R A C T
Consider a situation in which individuals -the buyers -have different valuations for the products of a given set. An envy-free assignment of product items to buyers requires that the items obtained by every buyer be purchased at a price not larger than his/her valuation, and each buyer's welfare (difference between product value and price) be the largest possible. Under this condition, the problem of finding prices maximizing the seller's revenue is known to be $\mathcal{A} \mathcal{P} \mathcal{X}$-hard even for unit-demand bidders (with several other inapproximability results for different variants), that is, when each buyer wishes to buy at most one item. Here, we focus on EnVy-Free Complete Allocation, the special case where a fixed number of copies of each product is available, each of the $n$ buyers must get exactly one product item, and all the products must be sold. This case is known to be solvable in $\mathrm{O}\left(n^{4}\right)$ time. We revisit a series of results on this problem and, answering a question found in Leonard (1983), show how to solve it in $\mathrm{O}\left(n^{3}\right)$ time by connections to perfect matchings and shortest paths.
\end{abstract}

(c) 2018 Elsevier B.V. All rights reserved.

\section{Introduction and background}

The purpose of the present note is to offer a refinement in a special case of a time-honored problem of economic theory, namely computation of Walrasian equilibrium, which also overlaps with the celebrated assignment problem of operations research.

Consider a set $B$ of $n$ buyers, each one interested in purchasing an item out a catalogue $C$ of $m \leq n$ products. Product $i$ is available in $c_{i}$ copies or items. Buyer $b$ has a valuation for each product, defined by a valuation function $v_{b}: C \rightarrow \mathbb{R}_{+}$, and all these functions are encoded into an $n \times n$ matrix $\mathbf{V}=\left[v_{b, i}\right]$.

We are interested in finding a complete allocation of items to buyers, i.e. a function $\phi: B \rightarrow C$, where we intend that buyer $b$ purchases product $\phi(b)$. With the term complete we mean that all the buyers must be satisfied, that is,

$$
n=\sum_{i \in C} c_{i} .
$$

Let $\Phi$ be the set of all complete allocations: for $\phi \in \Phi$ to be feasible, a price vector $\mathbf{p} \in \mathbb{R}_{+}^{m}$ must exist such that

(i) the price paid to any item never exceeds the buyer's valuation of the corresponding product:

$$
0 \leq p_{\phi(b)} \leq v_{b, \phi(b)} \quad b \in B,
$$

\footnotetext{
* Corresponding author.

E-mail addresses: claudio.arbib@univaq.it (C. Arbib), karasan@bilkent.edu.tr (O.E. Karaşan), mustafap@bilkent.edu.tr (M.Ç. Pınar).
} 
(ii) for each buyer, the difference between his/her valuation of the item bought and the price actually paid is the largest possible among all possible items:

$$
v_{b, \phi(b)}-p_{\phi(b)} \geq v_{b, i}-p_{i} \quad b \in B, i \in C .
$$

Price $p_{i}$ paid for product $i$ is non-discriminatory, meaning that different copies of the same product must be sold at the same price. Both $\phi$ and $\mathbf{p}$ fulfilling $(i)$ and $(i i)$ above are said to be envy-free. In a more general setting, where the number of buyers and product copies do not necessarily match, an envy-free pair $(\phi, \mathbf{p})$ is known as a Walrasian equilibrium; see $[9,10]$. From a seller's viewpoint, a problem arises of maximizing the revenue obtained. Note that in a Walrasian equilibrium, unsold items must have zero price. This is not the case in the general setting; see e.g., the discussion in [10]. In general, the seller's revenue does not coincide with the linear expression

$$
R(\mathbf{p})=\sum_{i \in C} c_{i} p_{i}
$$

unless (1) holds. Finding envy-free non-discriminatory prices (and a corresponding envy-free complete allocation) that maximize (4) is the ENVY-FREE COMPLETE ALLOCATION problem which is a special case of a problem studied since the early eighties to model elicitation of honest preferences [14], and later product-line positioning and pricing [5,6]. In the latter scenario, the so-called Profit Problem seeks to maximize the seller's revenue by deciding the products to offer and their allocation to buyers: when products are given, the model becomes the one addressed in the present paper. This model has also application in a facility location and pricing problem studied in [2]. There, a set of $m$ facilities offers service to a discrete set of $n \geq m$ clients, and client $k$ chooses the facility $i$ for which the total cost $p_{i}+d_{i}^{k}$ (price of the service + transportation cost to the facility) is minimum. The facility holder wants to choose prices $p_{i}$ so that the total revenue is maximized. If $\mathrm{s} / \mathrm{he}$ wants a solution where facility $i$ captures exactly $c_{i}$ clients, the objective reads exactly as (4). To transform the problem into ENVY-Free COMPLETE Allocation it suffices to identify clients with buyers, facilities with item types, and then choose $v_{b, i}=d-d_{i}^{b}$ as the valuation function of buyer $b$, where $d=\max _{i, k}\left\{d_{i}^{k}\right\}$.

Already in 1988, Dobson and Kalish [5] observed that when an allocation is given, finding the prices that maximize the seller's profit (the pricing problem) is the dual of a minimum cost flow problem. They take advantage of this observation to design a solution heuristic that moves from allocation to allocation, every time finding envy-free prices that, maximizing buyers' utility (value minus price, also referred to as welfare), make them happy with the products obtained. In [5] the authors compare different options, including a random choice, for the allocation to be chosen at each step of the algorithm and conclude that the best choice is one that maximizes buyers' welfare. The reasons are: maximum welfare is interesting by itself; moreover, since profit is part of the total welfare, the larger the latter the more profit can be carved (see [6], p. 167). While a negative-length cycle in the flow network developed in [5] and later used in [13,17] (a similar use of network flow construction is used in [17] in a somewhat different context of optimal auction design) is clearly addressed as the responsible of price infeasibility, no connection is apparently made between price infeasibility and welfare allocations: that is, which properties should an envy-free allocation have to guarantee the existence of prices fulfilling (i), (ii)? The question is answered in Section 3 of this paper as follows: an envy-free price vector for some allocation exists if and only if the allocation is a welfare maximizer. A subsequent question, which apparently remained unstudied, is also answered in the affirmative in Lemma 3 of the present paper: are welfare-maximizing allocations all equivalent from the viewpoint of the revenue that the corresponding envy-free prices guarantee to the seller? Finally, both [5,6] and [14] credit the pricing algorithm an $\mathrm{O}\left(n^{4}\right)$ complexity, and a call was made for a more efficient computational procedure (cf. section III, p. 472 of [14]). As we shall see in the sequel, the problem can in fact be solved in $\mathrm{O}\left(n^{3}\right)$.

\section{Envy-free perfect matching}

Without loss of generality, envy-free complete allocations can be reduced to permutations (or equivalently, perfect matchings). Recalling ( $i$ ), (ii) of Section 1, envy-free price vectors associated with a given $\phi$ must satisfy the following system of linear inequalities, cf. (2)-(3) introduced in the previous section:

$$
\begin{aligned}
& 0 \leq p_{\phi(b)} \leq v_{b, \phi(b)} \quad b \in B \\
& v_{b, \phi(b)}-p_{\phi(b)} \geq v_{b, i}-p_{i} \quad b \in B, i \in C .
\end{aligned}
$$

Let $N=\{1, \ldots, n\}$ denote the index set of both the buyers in $B$ and the copies of products (that is, the $n$ items) available. It is then easy to reduce $\phi$ to a permutation $\pi: N \rightarrow N$, where $\pi(i)$ is the index of the buyer that purchases item $i$. Instead of pricing products, we now price the $n$ individual items in $N$. An envy-free permutation, and the corresponding perfect matching

$$
M^{\pi}=\{(\pi(i), i): i \in N\}
$$

between buyers and items, is then one fulfilling

$$
\begin{aligned}
p_{i} & \leq v_{\pi(i), i} & & i \in N \\
p_{i}-p_{j} & \leq v_{\pi(i), i}-v_{\pi(i), j} & & i, j \in N \\
p_{i} & \geq 0 & & i \in N .
\end{aligned}
$$


The only question with (5)-(7) regards the assumption of non-discriminatory prices, as the system allows individual items to receive different prices. But suppose buyers $a, b$ get two copies $i, j$ of the same product. If $a$ is assigned $i$, then $p_{i}-p_{j} \leq v_{a, i}-v_{a, j}=0$, because $i$ and $j$ are identical items. Similarly, $p_{j}-p_{i} \leq v_{b, j}-v_{b, i}=0$. Therefore, $p_{i}=p_{j}$.

So let $\Pi$ be the set of all permutations of $N$ and $F R(\pi)=\left\{\mathbf{p} \in \mathbb{R}^{n}: \mathbf{p}\right.$ satisfies (5)-(7) $\}$ denote the feasible region of envy-free prices induced by $\pi$. Among all $\pi \in \Pi$, the seller seeks a revenue-maximizing one. In other words, from the seller's viewpoint, the problem is:

$$
\max _{\pi \in \Pi} \max _{\mathbf{p} \in F R(\pi)} \sum_{i=1}^{n} p_{i} .
$$

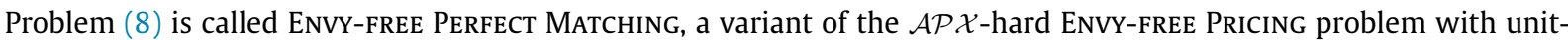
demand bidders where each buyer gets at most one object (see e.g. $[7,10,12,16]$ for a sample of the literature on envy-free pricing and connections to network pricing problems).

The $\mathcal{A P} \mathcal{X}$-Hardness result is from [10], but other approximation results (positive or negative) exist in the literature. A well studied problem is UniT-DEMANDED EnVY-Free Pricing, in which each consumer is willing (or is allowed) to buy at most one item, and there is an unlimited supply of each item: $(2 \log n)$ - and $(1+\varepsilon)$-approximation algorithms, where $n$ is the number of consumers, are respectively given in $[10,11]$, the second holding for a constant number of items. In [3] the hardness of approximation is analyzed in the case of uniform budget (equivalent to the min-buying problem with uniform budget proposed by [15]), where each consumer equally values all items s/he is interested in. Chalermsook et al. [4] studied the case in which the buyers have uniform budgets and are interested in at most $\ell$ items. They proved that approximating this problem is $\Omega\left(\ell^{1 / 2}\right)$-hard unless $P=N P$, thus the problem is not in $\mathcal{A P} \mathcal{X}$ unless $P=N P$. There are also other results using, for example, the Unique Games Conjecture and the Exponential Time Hypothesis; see references cited in [7] for a more complete and up-to-date review.

In ENVY-FREE PRICING, maximizing the sum of prices is in general not equivalent to maximizing the total revenue of the seller, because s/he can decide to remove from the market the items that force the solution to low prices. But in the special case considered here the two objectives are equivalent, and one can easily formulate it as a mixed-integer linear program: a permutation $\pi$ is encoded by $0-1$ decision variables $x_{b i}$, setting $x_{b i}=1$ if and only if $b=\pi(i)$ (or equivalently $(b, i) \in M^{\pi}$ ); then the problem is modeled as

$$
\max \sum_{i=1}^{n} p_{i}
$$

subject to

$$
\begin{array}{rlrl}
\sum_{i=1}^{n} x_{b i} & =1 & & b \in N \\
\sum_{b=1}^{n} x_{b i} & =1 & & i \in N \\
p_{i}-\sum_{b=1}^{n} v_{b, i} x_{b i} & \leq 0 & & i \in N \\
p_{i}-p_{j}-\sum_{b=1}^{n}\left(v_{b, i}-v_{b, j}\right) x_{b i} & \leq 0 & & i, j \in N \\
p_{i} & \geq 0 & & i \in N \\
x_{b i} & \in\{0,1\} & b \in N, i \in N .
\end{array}
$$

Objective function (9) represents the seller's total revenue from the sale of the objects. Eqs. (10)-(11) and domain restrictions (15) enforce that the $x$ variables encode a perfect matching, whereas inequalities (12)-(14) make the corresponding prices envy-free: in fact, by (11), one $x_{b i}$ only will get value 1 , and precisely the one corresponding to the client $b=\pi(i)$ that selects item $i$; consequently, the summations in (12), (13) return the right-hand sides of inequalities (5), (6), respectively.

EnVY-Free Perfect Matching is closely related to the well-known Perfect Matching problem. Here, we associate the former with this particular instance of the latter:

$$
\max _{\pi \in \Pi} \sum_{i=1}^{n} v_{\pi(i), i}
$$


which can be formulated:

$$
\max \sum_{b, i \in N} v_{b, i} x_{b i}
$$

subject to (10)-(11) and $x_{b i} \geq 0$. An optimal solution to this problem will be said $v$-maximum.

Let $z$ denote the optimal value of the above problem with all objects in $N$, and $z(j)$ denote the solution of the above problem with item $j$ removed from $N$, i.e., the optimal value of the bipartite matching problem:

$$
\max \sum_{b=1}^{n} \sum_{i \in N \backslash\langle j\}} v_{b, i} x_{b i}
$$

subject to

$$
\begin{array}{rlrl}
\sum_{i=1}^{n} x_{b i} \leq 1 & & b \in N \\
\sum_{b=1}^{n} x_{b i} & =1 & & i \in N \backslash\{j\} \\
x_{b i} & \geq 0 & & b \in N, i \in N .
\end{array}
$$

Then it is shown in [9] that the prices $p_{j}^{*}$ defined as $p_{j}^{*}=z-z(j)$ solve problem (8) (cf. Theorem 5 of [9]). Since the procedure requires the solution of $n+1$ linear programs of complexity $\mathrm{O}\left(n^{3}\right)$ each, the total complexity is $\mathrm{O}\left(n^{4}\right)$, the same indicated by the network flow procedure of $[5,6]$. We report in the next section a procedure which improves efficiency by an order of magnitude.

\section{Reduced problem complexity}

Definition 1. If $\pi(i)=i$ for every $i \in N$, then $\pi$ is termed identity-permutation.

Lemma 1. If $F R(\pi) \neq \emptyset$, then $\pi \in \Pi$ solves the PERFECT MATCHING problem (16).

Proof. Without loss of generality, suppose that $\pi$ is the identity-permutation (if not, rename items). Let $\mathbf{p} \in F R(\pi)$ and, indirectly, assume there exists $\gamma \in \Pi$ such that

$$
\sum_{i=1}^{n} v_{\gamma(i), i}>\sum_{i=1}^{n} v_{i, i} .
$$

Let $Q \subseteq N$ be such that $\gamma(i) \neq i$ for each $i \in Q$. Since $\gamma \neq \pi, Q$ is nonempty. In other words, inequality (18) reduces to

$$
\sum_{i \in Q} v_{\gamma(i), i}>\sum_{i \in Q} v_{i, i}
$$

As $\mathbf{p} \in F R(\pi)$, $\mathbf{p}$ fulfills inequalities (6), in particular those written for $i=\gamma(j), j \in Q$ :

$$
p_{\gamma(j)}-p_{j} \leq v_{\gamma(j), \gamma(j)}-v_{\gamma(j), j} \quad j \in Q .
$$

Since $\gamma(j) \in Q$ for each $j \in Q, \sum_{j \in Q} p_{\gamma(j)}-\sum_{j \in Q} p_{j}=0$. Thus, summing up the inequalities in (20) we get

$$
0 \leq \sum_{j \in Q} v_{\gamma(j), \gamma(j)}-\sum_{j \in Q} v_{\gamma(j), j},
$$

which contradicts (19).

We have just shown that feasible permutations, i.e. those leading to envy-free prices, are $v$-maximum. Let us now show that the converse of Lemma 1 is also true and conclude that the set of permutations encoding envy-free prices are simply the set of $v$-maximum perfect matchings. The analysis pertains to the question posed in Section 2: which properties should an envy-free allocation have to guarantee the existence of prices fulfilling $(i),(i i)$ ? The answer is that an envy-free price vector for some allocation exists if and only if the allocation is a welfare maximizer. To prove the result, for a given permutation $\pi$, we begin by constructing a weighted network $\left\langle G, \mathbf{w}^{\pi}\right\rangle$ with appropriate costs on arcs such that $F R(\pi)$ is non-empty if and only if shortest paths are well defined in $\left\langle G, \mathbf{w}^{\pi}\right\rangle$ as in [5,17]. In this construction, $G=\left(N \cup\{0\}, A=A_{0} \cup A_{1}\right)$ is a digraph where $A_{0}=\{(i, 0): i \in N\}$ and $A_{1}=\{(i, j): i \in N, j \in N \backslash\{i\}\}$.

Assign each $(i, 0) \in A_{0}$ the weight $w_{i 0}^{\pi}=v_{\pi(i), i}$, and each $(i, j) \in A_{1}$ the weight $w_{i j}^{\pi}=v_{\pi(i), i}-v_{\pi(i), j}$. With the weights so constructed, the shortest paths of $G$ into node 0 are well defined if and only if $G$ contains no negative-weight directed cycle. With this in mind, let us prove the following lemma. 
Lemma 2. If $\pi \in \Pi$ solves the Perfect Matching problem (16), then $F R(\pi) \neq \emptyset$.

Proof. Upon items renaming, suppose that the $\pi$ solving (16) is the identity-permutation. Let us show that $\left\langle G\right.$, $\left.\mathbf{w}^{\pi}\right\rangle$ has no cycle of negative weight. Indirectly, assume that $C=i_{1} \rightarrow i_{2} \rightarrow \cdots \rightarrow i_{t} \rightarrow i_{1}$ is a negative-weight cycle. Since no arcs leave node 0 , it follows $0 \notin C$. In other words,

$$
\sum_{(i, j) \in C} w_{i j}^{\pi}=\sum_{(i, j) \in C}\left(v_{i, i}-v_{i, j}\right)=\sum_{l=1}^{t} v_{i_{l}, i_{l}}-\sum_{l=1}^{t-1} v_{i_{l}, i_{l+1}}-v_{i_{t}, i_{1}}<0 .
$$

But the above inequality contradicts $\pi$ being $v$-maximum. Consequently, the shortest paths from each $i \in N$ to node 0 are well defined, and their lengths $p_{i}$ verify constraints (5)-(6), which correspond the optimality conditions for the shortest path problem; see [1]. To conclude $F R(\pi) \neq \varnothing$ it remains now to prove (7), that is, the lengths $p_{i}$ are non-negative. Let $P_{i} \subseteq A$ denote a generic $(i, 0)$-path, say $P_{i}=\left\{\left(i_{1}, i_{2}\right),\left(i_{2}, i_{3}\right) \ldots,\left(i_{t-1}, i_{t}\right)\right\}$ with $i_{1}=i$ and $i_{t}=0$. Its length is

$$
\sum_{a \in P_{i}} w_{a}^{\pi}=\sum_{l=1}^{t-1} v_{i_{l}, i_{l}}-\sum_{l=1}^{t-2} v_{i_{l}, i_{l+1}} \geq v_{i_{t-1}, i_{1}}
$$

since, again, $\pi$ is a $v$-maximum perfect matching. But $v_{i_{t}, i_{1}} \geq 0$ : hence $p_{i}$, the minimum of a set of non-negative path lengths, is itself non-negative.

After Lemma 2, to solve Envy-Free Perfect MAtching it is sufficient to consider optimal solutions to (16). The following result states that any two such solutions are equivalent in terms of the feasible regions (5)-(7) they induce.

Lemma 3. Let $\pi, \gamma \in \mathcal{P}$ be distinct permutations, both maximizing (16). Then, $F R(\pi)=F R(\gamma)$.

Proof. As in Lemma 1 , let $\pi$ be the identity-permutation and $\hat{\mathbf{p}} \in F R(\pi)$, that is:

$$
\begin{aligned}
\hat{p}_{i} & \leq v_{i, i} & & i \in N \\
\hat{p}_{i}-\hat{p}_{j} & \leq v_{i, i}-v_{i, j} & & i \in N, j \in N \backslash\{i\} \\
\hat{p}_{i} & \geq 0 & & i \in N .
\end{aligned}
$$

Let us show that $\hat{\mathbf{p}} \in F R(\gamma)$. For the converse, we can exchange the roles of $\pi$ and $\gamma$ and be done by symmetry.

For a given permutation $\alpha \in \Pi$, let us define a component as a subset of $N$, say $H$, such that for $i \in H, \alpha(i) \neq i$ and $\alpha(i) \in H$. Let $H_{1}, \ldots, H_{t}$ be such components for permutation $\gamma$. Since $\gamma \neq \pi, t \geq 1$.

Since both $\pi$ and $\gamma$ are $v$-maximum perfect matchings,

$$
\sum_{i \in H_{k}} v_{i, i}=\sum_{i \in H_{k}} v_{\gamma(i), i} \quad \text { for } k \in\{1, \ldots, t\} .
$$

In order to conclude that $\hat{\mathbf{p}} \in F R(\gamma)$, we need to show that (21)-(22) imply the consistency of the following system:

$$
\begin{aligned}
\hat{p}_{i} & \leq v_{\gamma(i), i} & & i \in N \\
\hat{p}_{i}-\hat{p}_{j} & \leq v_{\gamma(i), i}-v_{\gamma(i), j} & & i \in N, j \in N \backslash\{i\} .
\end{aligned}
$$

Let $\{r, s\}$ be an arbitrary distinct pair from $N$. To guarantee the consistency of the above system, it is enough to show that

$$
\begin{aligned}
\hat{p}_{r} & \leq v_{\gamma(r), r} \\
\hat{p}_{r}-\hat{p}_{s} & \leq v_{\gamma(r), r}-v_{\gamma(r), s} .
\end{aligned}
$$

If $\gamma(r)=r$, taking $i=r$ and $j=s$ in (21)-(22) will directly imply (25) and (26). So assume $r \in Q$ for some component $Q \in\left\{H_{1}, \ldots, H_{t}\right\}$. Without loss of generality, let $q=|Q|$ and $i_{1}, i_{2}, \ldots, i_{q}$ be an ordering of the elements in $Q$ such that $\left(i_{k}, i_{k+1}\right) \in M^{\gamma}$ for $k \in\{1, \ldots, q-1\}$ and $\left(i_{q}, i_{1}\right) \in M^{\gamma}$. Since $(i, i) \notin M^{\gamma}$ for any $i \in Q$ and $\gamma(i) \in Q$ for any $i \in Q$, such an ordering is readily available. Moreover, since the ordering is cyclical, it is possible to view $r$ as $i_{1}$, namely the first element in this ordering. Because $\hat{\mathbf{p}} \in F R(\pi)$, the following inequalities hold:

$$
\begin{aligned}
\hat{p}_{i_{1}}-\hat{p}_{i_{2}} & \leq v_{i_{1}, i_{1}}-v_{i_{1}, i_{2}} \\
\hat{p}_{i_{2}}-\hat{p}_{i_{3}} & \leq v_{i_{2}, i_{2}}-v_{i_{2}, i_{3}} \\
& \ldots \\
\hat{p}_{i_{q-1}}-\hat{p}_{i_{q}} & \leq v_{i_{q-1}, i_{q-1}}-v_{i_{q-1}, i_{q}} .
\end{aligned}
$$


Summing inequalities (27) we obtain

$$
\hat{p}_{i_{1}}-\hat{p}_{i_{q}} \leq \sum_{k=1}^{q-1} v_{k, k}-\sum_{k=1}^{q-1} v_{i_{k}, i_{k+1}} .
$$

As $\sum_{i \in Q} v_{i, i}=\sum_{i \in Q} v_{\gamma(i), i}$ by $(24)$, we have

$$
\hat{p}_{i_{1}}-\hat{p}_{i_{q}} \leq \sum_{k=1}^{q-1} v_{k, k}-\sum_{k=1}^{q-1} v_{i_{k}, i_{k+1}}=v_{i_{q}, i_{1}}-v_{i_{q}, i_{q}} .
$$

Writing (21) for $i=i_{q}$, we have

$$
\hat{p}_{i_{q}} \leq v_{i_{q}, i_{q}} .
$$

Finally, summing up (28) and (29), we conclude that

$$
\hat{p}_{i_{1}} \leq v_{i_{q}, i_{1}}=v_{\gamma\left(i_{1}\right), i_{1}}
$$

or that inequality (25) holds since $r=i_{1}$. Now we proceed to show that (26) holds. The case when $s=i_{q}$ is directly implied from (28). If $s \neq i_{q}$, applying (22) for $i=i_{q}$ and $j=s$, we have

$$
\hat{p}_{i_{q}}-\hat{p}_{s} \leq v_{i_{q}, i_{q}}-v_{i_{q}, s}
$$

Now, summing up (28) and (30) and substituting $r=i_{1}$ and $\gamma(r)=i_{q}$, we attain (26) as desired.

The above result answers 'yes' to the question: are welfare-maximizing allocations all equivalent from the viewpoint of the revenue that the corresponding envy-free prices guarantee to the seller? We can now derive the main result:

Theorem 1. EnVy-Free Perfect Matching can be solved in $O\left(n^{3}\right)$ time.

Proof. Lemmas 1-3 imply that in order to solve ENVY-FREE PERFECT MATCHING, it suffices to consider an arbitrary $v$-maximum perfect matching $\pi$. Since both Perfect Matching (17) and checking inequalities (5)-(7) boil down to a linear program, the problem belongs to the class $\mathcal{P}$. The best algorithm to solve weighted bipartite perfect matching uses a Fibonacci heap and has complexity $\mathrm{O}\left(n^{3}\right)$; [8]. On the other hand, as observed in Lemma 1, prices $p_{i}$ fulfilling (5)-(7) are computed by shortest $(i, 0)$-paths, which requires $\mathrm{O}\left(n^{3}\right)$ time with Bellman-Ford algorithm. The overall complexity follows.

\section{References}

[1] R.K. Ahuja, T.L. Magnanti, J.B. Orlin, Network Flows: Theory, Algorithms and Applications, Pearson, 1993.

[2] C. Arbib, M. Ç. Pınar, M. Tonelli, Stackelberg pricing in a one-dimensional model of duopoly with metric access costs. submitted for publication, 2017.

[3] P. Briest, Uniform budgets and the envy-free pricing problem, in: Proceedings of the 35th International Colloquium on Automata, Languages and Programming, 2008, pp. 808-819.

[4] P. Chalermsook, J. Chuzhoy, S. Kannan, S. Khanna, Improved hardness results for profit maximization pricing problems with unlimited supply, in: Proceedings of the 15th International Workshop, APPROX, and the 16th International Workshop, RANDOM, 2012, pp. 73-84.

[5] G. Dobson, S. Kalish, Positioning and pricing a product line, Mark. Sci. 7 (1988) 107-125.

[6] G. Dobson, S. Kalish, Heuristics for pricing and positioning a product-line using conjoint and cost data, Manage. Sci. 39 (1993) $160-175$.

[7] C.G. Fernandes, C.E. Ferreira, A.J.P. Franco, R.C.S. Schouery, The envy-free pricing problem, unit-demand markets and connections with the network pricing problem, Discrete Optim. 22 (2016) 141-161.

[8] M.L. Fredman, R.E. Tarjan, Fibonacci heaps and their uses in improved network optimization algorithms, J. ACM 34 (1987) 596-615.

[9] F. Gül, E. Stacchetti, Walrasian equilibrium with gross substitutes, J. Econom. Theory 87 (1999) 95-124.

[10] V. Guruswami, J.D. Hartline, A.R. Karlin, D. Kempe, C. Kenyon, F. McSherry, On profit-maximizing envy-free pricing, in: Proceedings of the sixteenth annual ACM-SIAM Symposium on Discrete Algorithms, 2005, pp. 1164-1173.

[11] J.D. Hartline, V. Koltun, Near-optimal pricing in near-linear time, in: Proceedings of the 9th Workshop on Algorithms and Data Structures, 2005, pp. 422-431.

[12] G. Heilporn, M. Labbé, P. Marcotte, G. Savard, A parallel between two classes of pricing problems in transportation and marketing, J. Revenue Pricing Manag. 9 (2009) 110-125.

[13] R. Kohli, R. Krishnamurti, Optimal product design using conjoint analysis: Computational complexity and algorithms, European J. Oper. Res. 40 (1989) $186-195$.

[14] H.B. Leonard, Elicitation of honest preferences for the assignment of individuals to positions, J. Polit. Econ. 91 (1983) 461-479.

[15] P. Rusmevichientong, B.V. Roy, P.W. Glynn, A nonparametric approach to multiproduct pricing, Oper. Res. 54 (1) (2006) 82-98.

[16] R. Shioda, L. Tunçel, T.G. Myklebust, Maximum utility product pricing models and algorithms based on reservation price, Comput. Optim. Appl. 48 (2011) 157-198.

[17] R.V. Vohra, Optimization and Mechanism Design, Math. Program. B 134 (2012) 283-303. 